The Importance of Experimental Design on Measurement of Dynamic Interfacial Tension and Interfacial Rheology in Diffusion-Limited Surfactant Systems

Matthew D. Reichert ${ }^{1}$, Nicolas J. Alvarez ${ }^{1}$, Carlton F. Brooks ${ }^{3}$, Anne M. Grillet ${ }^{3}$, Lisa A. Mondy ${ }^{3}$, Shelley L. Anna ${ }^{1,2}$, Lynn M. Walker ${ }^{1, *}$

1. Department of Chemical Engineering, Carnegie Mellon University, Pittsburgh, PA 15213

2. Department of Mechanical Engineering, Carnegie Mellon University, Pittsburgh, PA 15213

3. Sandia

Nat 


\title{
The Importance of Experimental Design on Measurement of Dynamic Interfacial Tension and Interfacial Rheology in Diffusion-Limited Surfactant Systems
}

\author{
Matthew D. Reichert ${ }^{1}$, Nicolas J. Alvarez ${ }^{1}$, Carlton F. Brooks ${ }^{3}$, Anne M. Grillet ${ }^{3}$, Lisa A. Mondy ${ }^{3}$, \\ Shelley L. Anna ${ }^{1,2}$, Lynn M. Walker ${ }^{1, *}$
}

1. Department of Chemical Engineering, Carnegie Mellon University, Pittsburgh, PA 15213

2. Department of Mechanical Engineering, Carnegie Mellon University, Pittsburgh, PA 15213

3. Sandia National Laboratories, Albuquerque, NM, 87123

\begin{abstract}
Pendant bubble and drop devices are invaluable tools in understanding surfactant behavior at fluid-fluid interfaces. The simple instrumentation and analysis are used widely to determine adsorption isotherms, transport parameters, and interfacial rheology. However, much of the analysis performed is developed for planar interfaces. The application of a planar analysis to drops and bubbles (curved interfaces) can lead to erroneous and unphysical results. We revisit this analysis for a well-studied surfactant system at air-water interfaces over a wide range of curvatures as applied to both expansion/contraction experiments and interfacial elasticity measurements. The impact of curvature and transport on measured properties is quantified and compared to other scaling relationships in the literature. The results provide tools to design interfacial experiments for accurate determination of isotherm, transport and elastic properties.

*corresponding author: lwalker@andrew.cmu.edu, (412) 268-3020

\section{Introduction}

Understanding the interfacial behavior of surfactants, or surface active agents, is vital to the design and optimization of commercial and consumer formulations involving these species. Interfacial behavior is critical to the performance of coatings, emulsions, oil dispersants, and even commercial food products. Generally, the first step of any interfacial study on surfactants is to define an equilibrium isotherm that describes the mass coverage of surfactant on an interface as a function of bulk concentration. This can be performed by measuring the interfacial tension, $\gamma$, of a surfactant solution in contact with some other immiscible fluid phase (air, water or oil) for a range of surfactant concentrations. The interfacial tension, $\gamma$, is related to the mass coverage, $\Gamma$, via the Gibbs equation, $\Gamma=d \gamma / d \ln (C)$, where $C$ is the bulk concentration.
\end{abstract}


ional Laboratories, Albuquerque, NM, 87123

\begin{abstract}
Pendant bubble and drop devices are invaluable tools in understanding surfactant behavior at fluid-fluid interfaces. The simple instrumentation and analysis are used widely to determine adsorption isotherms, transport parameters, and interfacial rheology. However, much of the analysis performed is developed for planar interfaces. The application of a planar analysis to drops and bubbles (curved interfaces) can lead to erroneous and unphysical results. We revisit this analysis for a well-studied surfactant system at air-water interfaces over a wide range of curvatures as applied to both expansion/contraction experiments and interfacial elasticity measurements. The impact of curvature and transport on measured properties is quantified and compared to other scaling relationships in the literature. The results provide tools to design interfacial experiments for accurate determination of isotherm, transport and elastic properties.
\end{abstract}

*corresponding author: lwalker@andrew.cmu.edu, (412) 268-3020

\title{
Introduction
}

Understanding the interfacial behavior of surfactants, or surface active agents, is vital to the design and optimization of commercial and consumer formulations involving these species. Interfacial behavior is critical to the performance of coatings, emulsions, oil dispersants, and even commercial food products. Generally, the first step of any interfacial study on surfactants is to define an equilibrium isotherm that describes the mass coverage of surfactant on an interface as a function of bulk concentration. This can be performed by measuring the interfacial tension, $\gamma$, of a surfactant solution in contact with some other immiscible fluid phase (air, water or oil) for a range of surfactant concentrations. The interfacial tension, $\gamma$, is related to the mass coverage, $\Gamma$, via the Gibbs equation, $\Gamma=d \gamma / d \ln (C)$, where $C$ is the bulk concentration.

For interfaces not at equilibrium, understanding of specific interfacial behavior requires characterization of the transport dynamics of surfactant to the interface. An equilibrium isotherm, combined with an equation of state (which relates the interfacial tension to the interfacial coverage), 
allows quantification of changes in interfacial coverage that arise either from normal equilibration processes (such as transport to an initially surfactant-free interface), or forced changes in interfacial area. Both the isotherm and mass transport problems are often complex and the goal of experiments is to parameterize the results with respect to a transport model. The isotherm and equation of state contain parameters including a maximum packing concentration, ratios of adsorption/desorption probabilities and other terms to capture intermolecular interactions and packing on the interface. Mass transport models include bulk diffusion coefficients, specific rate constants for adsorption or desorption, local flow or convection and geometry and incorporation of the isotherm as a boundary condition.

Digital imaging methods have allowed pendant bubble and pendant drop techniques to become core tools in the characterization of interfacial surfactant dynamics. ${ }^{[1]}$ These techniques consist of forming a millimeter scale droplet or bubble pinned to the end of a capillary immersed in a volume of surfactant solution. Shape changes in the interface, which is distended by gravity or buoyancy, are analyzed and focus has been on the details of the fitting algorithms. ${ }^{[2-4]}$ Since surfactant behavior is sensitive to the interfacial concentration, the contraction or expansion of an interface is used for both determining transport parameters and verifying equilibrium isotherm parameters. ${ }^{[5-9]}$ A pendant bubble or drop is allowed to equilibrate, and is then compressed or expanded at a constant rate to extract isotherm parameters. As techniques using milliscale and microscale interfaces become more common, it is important to assess the relative impact of isotherm and transport properties on the measured interfacial/surface tension.

Surfactant adsorption to the interface is governed by diffusion and "reaction", or adsorption/desorption, kinetics. ${ }^{[10-12]}$ The scope of this study is focused on diffusion-limited systems where adsorption kinetics is assumed to not impact the observed behavior, and more specifically addresses the impact of interfacial curvature. And while discussions of curvature effects have long been present in the literature, their adaptation has been limited. ${ }^{[13,14]}$ In a diffusion-limited surfactant system, the bulk concentration near the interface is assumed to be in equilibrium with the interface at all times. Applied surface deformations change the interfacial concentration, which simultaneously changes the concentration near the interface to maintain equilibrium. Concentration gradients between the fluid near the interface and the bulk then drive diffusion of molecules to or away from the interface. 
The surface coverage of surfactant as a function of time is described with Fick's law of diffusion, in spherical coordinates,

$$
\frac{\partial C}{\partial t}+v_{r} \frac{\partial C}{\partial r}=D \frac{1}{r^{2}} \frac{\partial}{\partial r}\left(r^{2} \frac{\partial C}{\partial r}\right)
$$

Where $v_{r}$ is the fluid radial velocity, $\mathrm{r}$ is the radius, $\mathrm{t}$ is time, $\mathrm{D}$ is the bulk diffusion coefficient, and $C(r, t)$ is the concentration as a function of radius and time. The use of this equation to model mass transport to a curved interface is well established ${ }^{[15,16]}$ in cases where the Peclet number is much greater than one, a planar assumption holds where the transport lengthscale is that of the fluid boundary layer. We will show that even in cases of high $P e$ cases for compression and expansion, it is still possible to need to account for curvature in elasticity measurements.

The mass balance at the interface, again in spherical coordinates, is given as

$$
\frac{d \Gamma}{d t}+\frac{2 \Gamma}{r} \frac{d r}{d t}=D\left(\frac{d C}{d r}\right)_{r=R}
$$

An isotherm is needed to define $\Gamma(\mathrm{C})$ and for this study, the Generalized Frumkin isotherm is used, 


$$
\frac{\Gamma}{\Gamma_{\infty}}=x=\frac{C}{a \cdot \exp K x^{n}+C},
$$

as it describes the behavior of surfactant used in this work. ${ }^{[1,17-19]}$ The parameters that are specific to the surfactant are D (the molecular diffusion coefficient) and the four isotherm parameters, $K$, which represents a surface van der Waals interaction term, $n$, a fitting parameter that adjusts the isotherm between the Frumkin $(\mathrm{n}=1)$ and Langmuir $(\mathrm{n}=0)$ equations, $a$, the ratio of the desorption to adsorption kinetic rate constants and, $\Gamma_{\infty}$, the surface coverage at its maximum value. ${ }^{[20]}$ Note that none of these parameters should depend on the bulk concentration or the geometry of the interface. We define $\mathrm{x}=\Gamma /$ $\Gamma_{\infty}$ for convenience. The Gibbs equation using the Generalized Frumkin isotherm provides an equation of state to relate the measurable interfacial or surface tension to interfacial concentration,

$$
\gamma-\gamma_{0}=\Gamma_{\infty} R T\left[\ln (1-x)-\frac{K n}{n+1} x^{n+1}\right] .
$$

Solving equations (1)-(4) allows the measurable dynamic interfacial tension to be related to the transport in these complex systems. This combination of the diffusion equation and isotherm provides a model that is used to predict the interfacial properties as a function of time or change in the interfacial area given the isotherm and transport parameters.

Dilatational elasticity measurements also provide a tool to determine mass transport properties of surfactants. Using pendant bubble or drop tensiometry, the interfacial response to an oscillatory area deformation is used to approximate dilatational rheology, ${ }^{[21-25]}$ and then extract diffusion coefficients from model fits to data. This is achieved by measuring the surface tension of a drop or bubble during an applied deformation, and characterizing the response as a function of deformation rate and the equation of state for the surfactant in question. The ratio of the response to the deformation is the complex modulus $E^{*}$, where the real and imaginary parts correspond to an elastic and viscous contribution. Using the Fourier transform of the deformation, it is possible to calculate $\mathrm{E}^{*},{ }^{[5]}$

$$
E^{*}(\omega)=\frac{\mathcal{F}\{\Delta \gamma(t)\}}{\mathcal{F}\{\Delta \ln (A(t))\}},
$$


where $\mathrm{F}$ is the Fourier transform function, $\Delta \gamma(\mathrm{t})$ is the change in surface tension as a function of time, $\mathrm{A}(\mathrm{t})$ is the surface area as a function of time, and $\mathrm{E}^{*}$ is a function of angular frequency $\omega$. For a smallamplitude single harmonic (i.e., sine wave) oscillation of the interfacial area, the modulus becomes,

$$
E^{*} \approx A_{0} \frac{d \gamma}{d A}
$$

where $d \gamma$ is the amplitude of the surface tension signal, $d A$ is equal to the amplitude of the area signal, and $\mathrm{A}_{0}$ is the median area of the applied oscillation. The origin of this stress-strain relationship is the focus of many publications. ${ }^{[9,26-32]}$

For the simplest case of no mass transfer between the interface and the bulk and no interaction between adsorbed molecules on the interface, the elasticity is constant over small deformations, and is referred to as the Gibbs elasticity ${ }^{[33]}$

$$
E_{0}=-\frac{d \gamma}{d \ln (\Gamma)}
$$

where the Gibbs elasticity is calculated from the equation of state of the surfactant that relates surface coverage $\Gamma$ to surface tension $\gamma$, for example the expression given in Equation (4). In Gibbs' original derivation of this quantity for experiments with surfactant-coated films, ${ }^{[34]}$ it was assumed that there was no mass transfer between the bulk and interface and the elasticity is given as $E=d \gamma / d \ln (A)$. Equation (7) is the result of this assumption and is referred to as the Gibbs modulus. For cases where mass transfer between the interface and the bulk contributes to the measured elasticity, $E^{*}(\omega)$ is given in Equation (5) and will have more complicated frequency dependence providing a probe of transport phenomena.

In many cases, oscillatory deformation is used to probe elasticity by fitting a sine wave to the surface area deformation and surface tension response to determine the elasticity. ${ }^{[8,21,23-25,29]}$ For small amplitude oscillatory deformations, the following analytical solutions are outlined in detail in the literature and we repeat them here for reference. ${ }^{[25,32,35]}$

For diffusion-limited transport to a planar interface and independent of a specific isotherm, the complex elastic modulus is given by ${ }^{[32,35]}$ 


$$
E^{*}=\frac{E_{0}}{1+\left(\frac{\omega_{L V}}{i \omega}\right)^{1 / 2}},
$$

where $\omega_{L V}$ is the natural frequency of the system given by

$$
\omega_{L V}=D\left(\frac{d C}{d \Gamma}\right)^{2} .
$$

In Equations (8) and (9), $\omega$ is the frequency of the oscillation of the interfacial area, and $\mathrm{dC} / \mathrm{d} \Gamma$ is determined from the surfactant response to the change in the interfacial area through the isotherm. Because Equation (8) is complex, the real ( $\left.E^{\prime}\right)$ and imaginary $\left(E^{\prime \prime}\right)$ components are often separated, and an effective surface dilatational viscosity $\left(\eta_{\mathrm{s}}\right)$ is calculated by assuming that $E^{\prime \prime}=\omega \eta_{s} \cdot{ }^{[21]}$ Since complexities arise in the separation of in- and out-of-phase components of a sinusoidally varying signal, we will report $E^{*}(\omega)$ and compare to predictions of the transport model. At high frequencies, the modulus will limit to the Gibbs modulus while at low frequencies the modulus will be dominated by transport and limit to a power law dependence on frequency. For the planar system, the natural frequency $\omega_{\mathrm{LV}}$ will appear as a turnover frequency, $\omega_{\mathrm{T}}$, between these two limiting behaviors. Note that the turnover frequency will depend on the diffusion coefficient and the details of the isotherm. This expression was shown to describe data collected using a Langmuir trough with an oscillating barrier for decanoic acid. ${ }^{[32]}$ Equation (9) has been routinely applied with mixed success. ${ }^{[9,36-39]}$ Application of these expressions to systems with curvature (pendant drop and bubble) must be done with care.

For diffusion-limited transport to a spherical interface, the value of the turnover frequency is a function of the radius of curvature of the interface, due to the impact of curvature on mass transport. For a pendant drop, where surfactant adsorbs from the inside of the drop to the interface, the elasticity, $E_{D}{ }^{*}$, is given by ${ }^{[35]}$

$$
\frac{E_{D} *}{E_{0}}=\frac{1}{1+\frac{D}{i \omega R} \frac{d C}{d \Gamma}\left(\sqrt{\frac{i \omega}{D}} R \operatorname{coth}\left(\sqrt{\frac{i \omega}{D}} R\right)-1\right)}
$$


where $\mathrm{R}$ is the radius of the drop. For diffusion of surfactant to a bubble from an external bulk solution, the solution for the elasticity, $\mathrm{E}_{\mathrm{B}}{ }^{*}$, takes the form,

$$
\frac{E_{B}^{*}}{E_{0}}=\frac{1}{1+\frac{D}{i \omega R} \frac{d C}{d \Gamma}\left(1+\sqrt{\frac{i \omega}{D}} R\right)} .
$$

Equations (8), (10) and (11) provide different functional forms for the frequency dependence of the elasticity of an interface, and should be used appropriately, based on the isotherm properties, radius of curvature and transport properties of the surfactant.

Here, we revisit the transport of a well-characterized surfactant to air-water interfaces. The theory and modeling outlined above is applied and comparisons are made to a recent scaling developed for diffusion-limited adsorption to curved interfaces. Practical issues associated with extracting isotherm and transport parameters from expansion/contraction experiments as well as interfacial rheology are discussed. This work also provides simple tools to determine the impact of curvature effects.

\section{Materials and Methods}

The surfactant used in this study is dodecyl octaethylene glycol ether $\left(\mathrm{C}_{12} \mathrm{E}_{8}\right)$. With the exception of the pendant drop experiments, $\mathrm{C}_{12} \mathrm{E}_{8}$ is purchased from Nikko Chemical Co. (Tokyo, Japan) at $99 \%$ purity, and solutions are prepared with ultrapure deionized water (resistivity $18.2 \mathrm{M} \Omega \cdot \mathrm{cm}$ ) from a Barnstead water purification system. For pendant drop experiments, $\mathrm{C}_{12} \mathrm{E}_{8}$ is purchased at $98 \%$ purity from Sigma. This difference does not affect the results.

The parameters used for the Generalized Frumkin model (Equation 3) are $\Gamma_{\infty}=5.3 \times 10^{-6} \mathrm{~mol} / \mathrm{m}^{2}$, $\mathrm{a}=2.3 \times 10^{-6} \mathrm{~mol} / \mathrm{m}^{3}, \mathrm{~K}=13.23$, and $\mathrm{n}=0.5$. The value of the diffusion coefficient, $\mathrm{D}$, for $\mathrm{C}_{12} \mathrm{E}_{8}$ in dilute solution is given as $\mathrm{D}=3.8 \times 10^{-10} \mathrm{~m}^{2} / \mathrm{s}$. These values have been used in several previous publications for this surfactant. ${ }^{[17,20,26]}$

Pendant drop and bubble experiments are performed using a Newport LTA Series motorized actuator connected to a Hamilton gastight syringe, suspended above a $2 \mathrm{~mL}$ cuvette cell. The cell is capped with a lid and fitted with a hole to allow passage of the needle. Drops are formed at the end of a 
PTFE coated needle, and are monitored with a Basler A622F-1394 camera with an Edmund Optics VZM 450 lens. For each experiment, the solution is first forced into the cell to create a humid environment, and temperature control is achieved with a coil of Tygon ${ }^{\circledR}$ tubing wrapped at the base of the cell fed by a temperature bath. Temperature is monitored with a thermocouple held in the cell through a second hole in the cell cap. Drop equilibration requires between one and three days depending on the bulk concentration; long equilibration times are due to significant depletion of surfactant in the confined volume of the syringe, thus limiting the flux of material to the interface.

After equilibration, different deformations (constant-rate compression, constant-rate expansion, or oscillatory) are performed by drawing on the syringe with the actuator at a set rate or frequency. Images are analyzed in Labview ${ }^{\circledR}$ by fitting the drop shape to a Bond number, ${ }^{[4]}$ resulting in an error of about $\pm 0.25 \mathrm{mN} / \mathrm{m}$. Pendant bubble compressions and expansions are performed in a similar manner, with the exception that a J-hook PTFE needle was used, and a Hamilton syringe pump drove the deformation. Bubbles are formed in a $15 \mathrm{~mL}$ cell to minimize surfactant depletion to the container. Equilibration for pendant bubble tests takes approximately $20-30$ minutes.

To measure properties of micron-scale bubbles, a microtensiometer is used. ${ }^{[18,19,40,41]}$ The microtensiometer consists of a capillary needle immersed in solution, contained in a holding cell molded from polydimethylsiloxane (PDMS). The pressure of the air bubble at the tip of the needle is measured by an Omega Model PX409-001GV pressure transducer, having a sensitivity of $\sim 10$ Pa and maximum gauge pressure limit of $6000 \mathrm{~Pa}$. This pressure is set by a water column of known height in line with the air phase. To calculate the pressure jump across the interface, $\Delta \mathrm{P}$, the hydrostatic pressure resulting from the fluid in the sample cell is subtracted from the transducer pressure. The capillary tip is observed through a Nikon T-300 inverted light microscope, and images are collected using a Diagnostic Instruments Spot RT Monochrome digital camera. The radius of the bubble is fit with an image analysis code developed in LabView ${ }^{\circledR}$. Using the Young-Laplace equation, $\gamma=\Delta P R / 2$, the surface tension is measured during the course of an experiment. To oscillate the pressure head for interfacial rheology experiments, a brushless DC motor is added at the base of the column that drives a syringe in an oscillatory fashion. The syringe injects and removes water from the column through a three-way valve, creating an oscillating pressure head. ${ }^{[30]}$ This results in an oscillatory deformation of the air-water 
interface at the tip of the capillary. Using the equation for the area of a spherical cap, $A=\pi\left(R_{c}{ }^{2}+h^{2}\right)$, where $R_{c}$ is the capillary radius and $h$ is the distance between the center of the surface perpendicular to the end of the capillary and the tip of the bubble, a sinusoidal deformation is calculated. For all oscillatory data, Equation (6) is used to determine the complex elasticity.

The model for a constant rate of deformation is calculated using Equations (1)-(3), with the boundary conditions for a pendant bubble experiment (infinitely far from the interface the bulk concentration remains constant for all times). Since Equation (3) is nonlinear, the analysis results in an integral that is evaluated numerically. For this, the time variable was discretized using a first-order Euler approximation and the remaining integral was solved using a trapezoidal discrete integration scheme in Matlab $^{\circledR}$. The mass balance in Equation (2) is iterated until it satisfies an error tolerance of $0.005 \%$ of the difference between the surface and local bulk concentration. The surface coverage response to deformation is then used in Equation (4) to calculate a surface tension response. Input parameters include surfactant concentration, a rate of deformation, and an initial bubble radius. To approximate the planar case, an artificially large bubble radius is used, a value that results in a limit to the planar case. Oscillatory deformations are also modeled using this code, by altering the constant rate of deformation to a sine or cosine deformation.

\section{Results}

Bubble expansion experiments are performed on a pendant bubble to characterize the interfacial response to a controlled change in area. Experimental results are compared to the predictions (Eq (1)-(4)) of the dynamic surface tension as each interface undergoes an expansion using the planar model. Experiments are performed on a typical bubble $(\mathrm{R}=1 \mathrm{~mm})$ equilibrated in a $6.25 \mu \mathrm{M}$ solution of $\mathrm{C}_{12} \mathrm{E}_{8}$. 


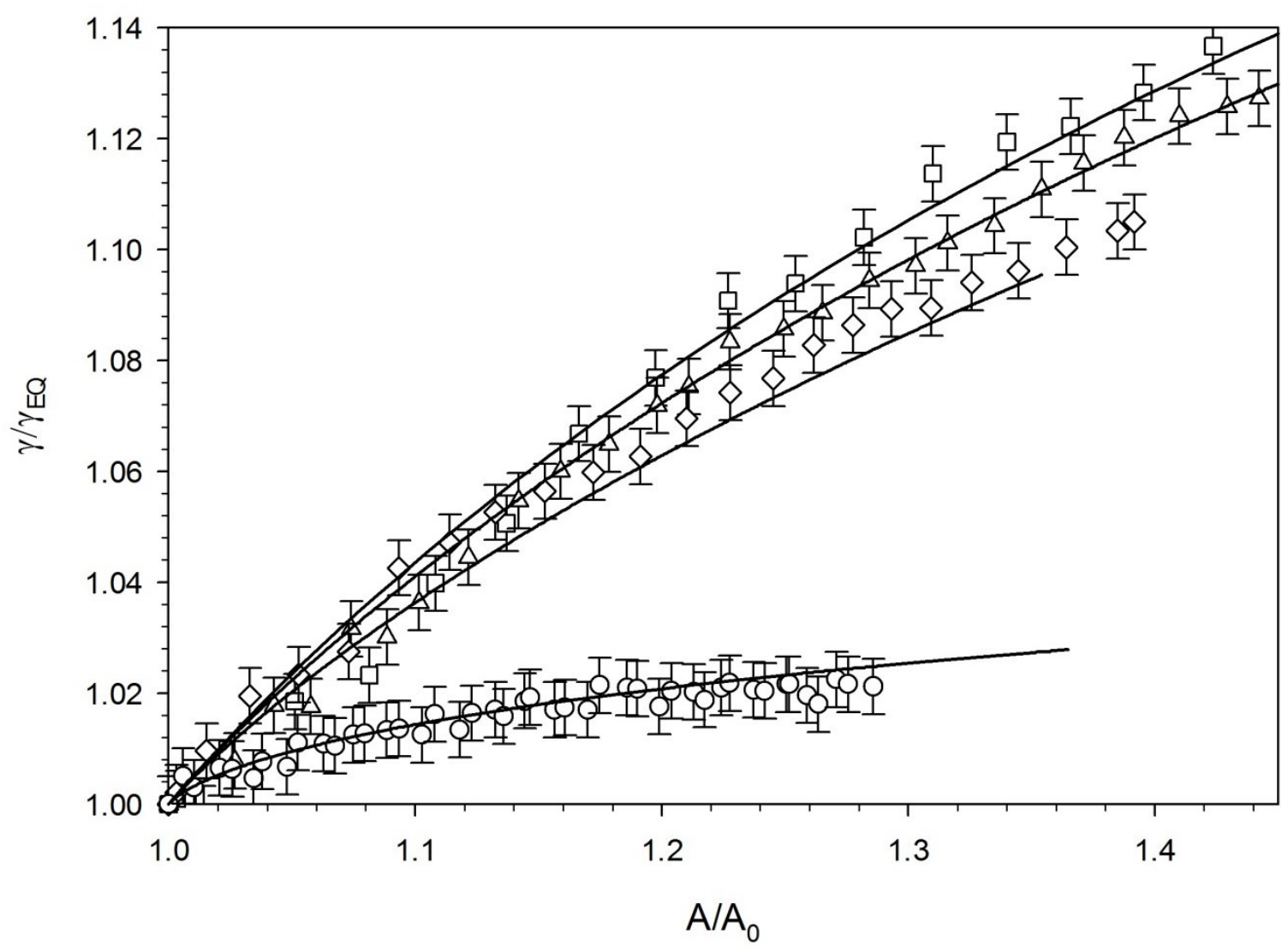

Figure 1. Pendant bubble expansion experiments, showing $\gamma / \gamma_{\mathrm{EQ}}$ versus $\mathrm{A} / \mathrm{A}_{0}$ for $\mathrm{C}=6.25 \mu \mathrm{M} \mathrm{C}_{12} \mathrm{E}_{8}$, with $\mathrm{R}_{\mathrm{b}}=1 \mathrm{~mm}$. Expansion rates $\left(1 / \mathrm{A}_{0}\right)(\mathrm{dA} / \mathrm{dt})$ are $3 \times 10^{-1} \mathrm{~s}^{-1}(\square), 1 \times 10^{-1} \mathrm{~s}^{-1}(\triangle), 1 \times 10^{-2} \mathrm{~s}^{-1}(\diamond)$, and $1 \mathrm{x}$ $10^{-3} \mathrm{~s}^{-1}(\mathrm{O})$. Solid lines are model predictions assuming a planar interface and the same concentration, radius, and expansion rate for each set of data.

Figure 1 shows the normalized surface tension as a function of area for the air-water interface. Symbols denote experimental data while the solid lines are the predictions. The dynamic surface tension is normalized by the initial equilibrium value, $\gamma_{\mathrm{EQ}}=51.4 \mathrm{mN} / \mathrm{m}$, and the area is normalized by the initial area of the bubble. As time increases during the expansion, the surface tension increases because the area is increasing, causing a reduction in interfacial concentration. At lower expansion rates, diffusion to the interface allows the system to adjust the interfacial concentration causing the deviation from the initial value to be smaller. The slowest rate, $1 \times 10^{-3} \mathrm{~s}^{-1}$, shows only a $2 \%$ increase in surface tension through a 
$30 \%$ increase in area because surfactant has time to re-populate the interface in response to the slow area increase. The model predictions agree well with experimental data with no adjustable parameters.

For the model predictions, a planar geometry is assumed, as is often done in the analysis of similar experimental results. The Peclet number $(\mathrm{Pe}=\mathrm{UR} / \mathrm{D})$ ranges from 400 in the most rapid expansion to $\sim 1$ for the slowest expansion, based on $R=1 \mathrm{~mm}$ and $\mathrm{U}=0.15 \mathrm{~mm} / \mathrm{sec}$ for the most rapid expansion. While convection (which is accounted for in the model) may impact transport of material to the interface in the fastest case, in the slowest response, it is unlikely. For the slowest expansion, mass transport and deformation timescales are similar, and convection does not justify the reduction in the rate of change of $\gamma / \gamma_{\mathrm{EQ}}$ that is observed.

The pendant drop case is studied in a similar manner. Because pendant drop experiments often demonstrate significant surfactant depletion of the bulk concentration, model calculations are performed by setting the initial drop concentration to the value corresponding to the measured surface tension observed in experiment, and not necessarily that of the concentration used in sample preparation. This approach is typically used in the analysis of depleted systems. ${ }^{[40,42,43]}$ In this case, the surface tension equilibrates at $51.6 \pm 0.3 \mathrm{mN} / \mathrm{m}$, consistent with a bulk concentration of $6.0 \mu \mathrm{M}$ as measured prior to any change in the area. This is a lower concentration than the preparation concentration of $6.25 \mu \mathrm{M}$. 


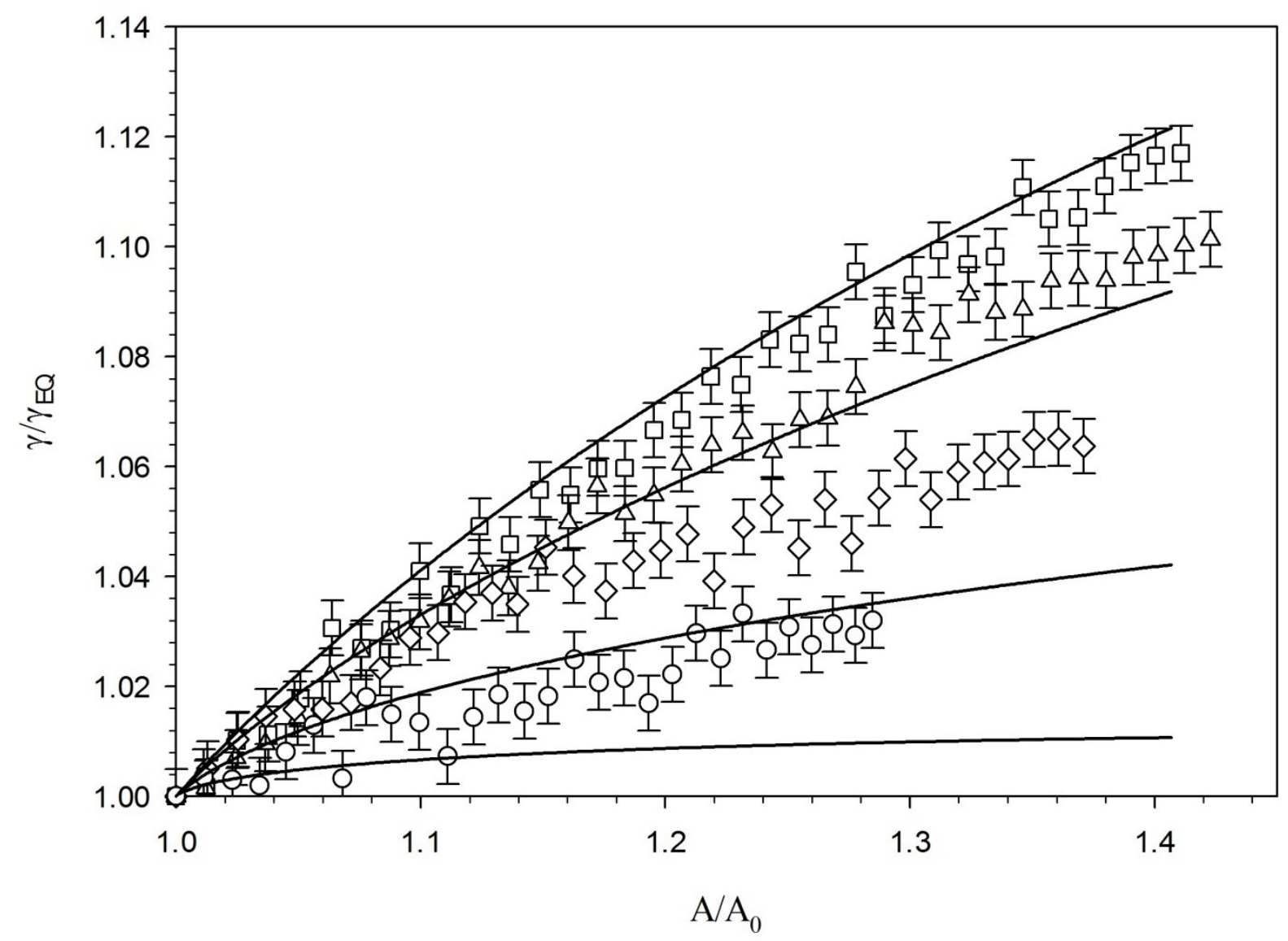

Figure 2. Pendant drop expansion experiments, showing $\gamma / \gamma_{\mathrm{EQ}}$ versus $\mathrm{A} / \mathrm{A}_{0}$ for a bulk concentration (based on measured $\gamma$ ) of $\mathrm{C}=6.0 \mu \mathrm{M} \mathrm{C}_{12} \mathrm{E}_{8}$, with $\mathrm{R}_{\mathrm{d}}=1 \mathrm{~mm}$. Expansion rates $\left(1 / \mathrm{A}_{0}\right)(\mathrm{dA} / \mathrm{dt})$ of $2 \times 10^{-1} \mathrm{~s}^{-1}$ $(\square), 2 \times 10^{-2} \mathrm{~s}^{-1}(\triangle), 2 \times 10^{-3} \mathrm{~s}^{-1}(\diamond)$, and $2 \times 10^{-4} \mathrm{~s}^{-1}(\bigcirc)$. Solid lines are model predictions using the same concentration, radius, and expansion rate for each set of data.

Figure 2 shows the results of drop expansions. Again, the surface tension is normalized by the initial value, or $51.6 \mathrm{mN} / \mathrm{m}$, and it is plotted as a function of the change in area, $\mathrm{A} / \mathrm{A}_{0}$. For each set of data, the normalized surface tension increases as the drop expands. Model predictions are also performed assuming a planar geometry. The model curves increase in a qualitatively similar manner to the data, but do not agree with the data for all experiments. The smaller the expansion rate, the greater the model underpredicts the data. At the slowest expansion rate of $2 \times 10^{-4} \mathrm{~s}^{-1}$, the data indicates a value of $\gamma / \gamma_{\mathrm{EQ}}$ of 
1.03 at a $30 \%$ increase in the area, while the prediction indicates a value of $\gamma / \gamma_{\mathrm{EQ}}$ of 1.01 , corresponding to a $1 \mathrm{mN} / \mathrm{m}$ difference in surface tension. These differences arise from the fact that the drop does not have the same curvature as a planar interface. The lengthscale of diffusion becomes limited by the radius of the droplet and hinders transport. This allows for larger perturbations from equilibrium mass coverage of the interface for expanding droplets. These differences are more pronounced at lower deformation rates, as the timescales for transport to the interface begin to balance with the timescale of deformation.

Oscillating the area of the interface to probe elasticity more dramatically demonstrates the effect that geometry has on surfactant transport during elasticity measurements. Elasticity measurements are performed using the same drop system presented in Figure 2 by oscillating about a median area for a range of frequencies, and the complex modulus is calculated using Equation (6). Additionally, oscillatory experiments are performed on the microtensiometer using a $6.0 \mu \mathrm{M}$ solution of $\mathrm{C}_{12} \mathrm{E}_{8}$ with a $30 \mu \mathrm{m}$ radius capillary. Model predictions using Equations (8), (10), and (11) for the bubble, drop, and planar case, using a $1 \mathrm{~mm}$ radius for the drop and a $30 \mu \mathrm{m}$ radius for the bubble, and at a bulk concentration of $6.0 \mu \mathrm{M}$ $\mathrm{C}_{12} \mathrm{E}_{8}$ are also shown. Area amplitudes of the oscillation for each experiment range from $3-5 \%$ and results are shown in Figure 3. 


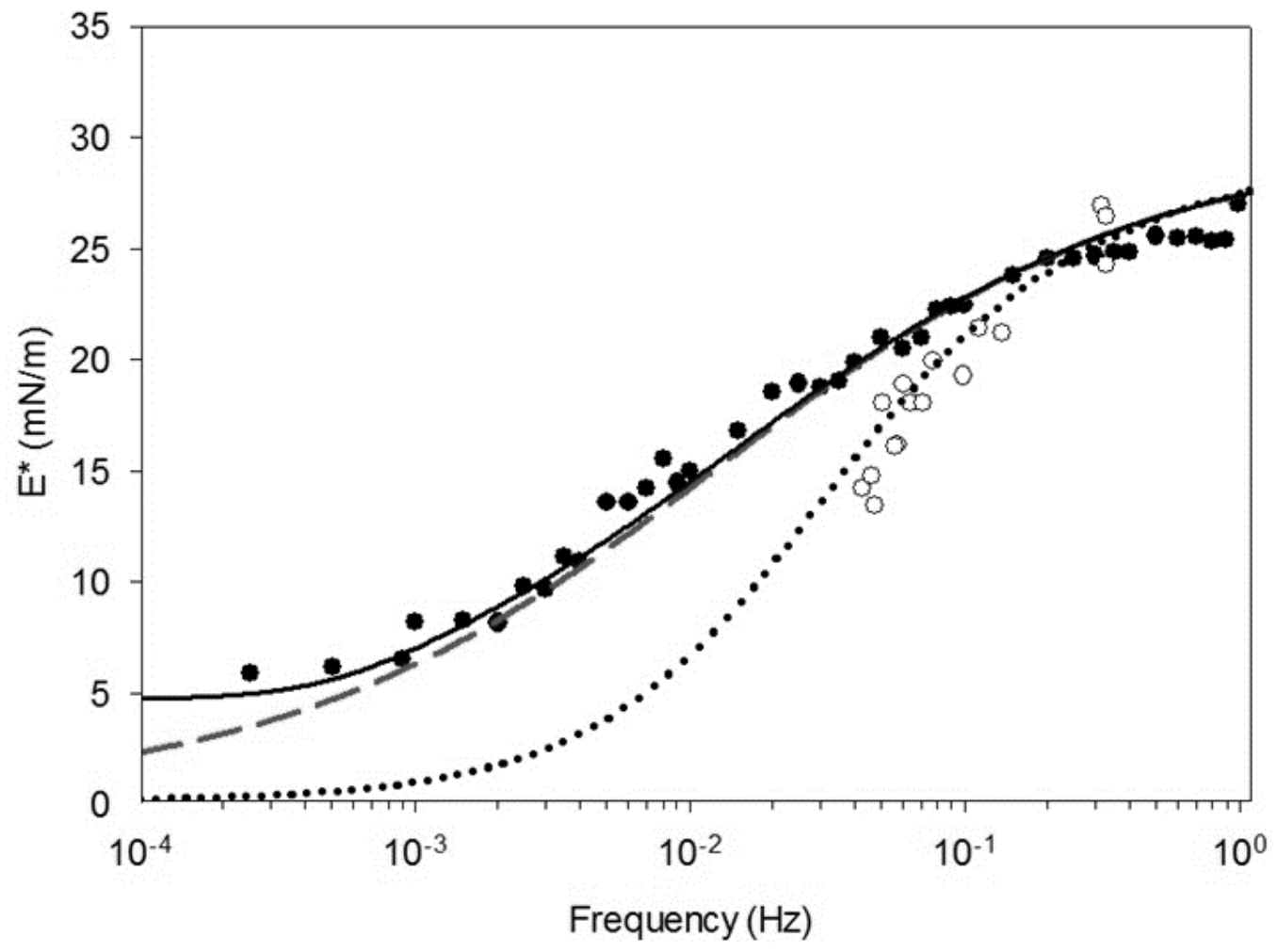

Figure 3. Complex modulus, $E^{*}$, versus frequency, $\omega$, for pendant drop $\left(\bullet, R_{d}=1 \mathrm{~mm}\right)$, microtensiometer $\left(\circ, R_{b}=30 \mu \mathrm{m}\right)$, and calculations using the planar (dashed line), drop (solid line), and bubble (dotted line) geometries. $\mathrm{C}=6.0 \mu \mathrm{M}$ for all cases.

In Figure 3, the elasticity is plotted as a function of frequency for two sets of experimental data and three model predictions. For the two sets of experimental measurements of $E^{*}(\omega)$, the modulus shows frequency dependence over the range probed. There appears to be a low frequency plateau of about $5 \mathrm{mN} / \mathrm{m}$ for the pendant drop data. Both sets of experimental data rise throughout the measured frequency range and approach the Gibbs modulus of $30 \mathrm{mN} / \mathrm{m}$ at high frequency. The microtensiometer, with a higher radius of curvature, obtains a lower value for the modulus at all frequencies. The model predictions for both the drop and bubble case agree with the experimental data. The pendant drop data compares well with the drop calculations and also to the planar predictions due both to the relatively large radius of this drop and the bulk surfactant concentration. The deviation at low frequency is due to conservation of mass in a finite drop and will be discussed later. $E^{*}(\omega)$ measured on a $30 \mu \mathrm{m}$ bubble is 
generally lower than the planar case, but again both models and data converge at high frequency, close to the Gibbs elasticity limit. This highlights the fact that low frequency oscillations are useful for probing transport mechanisms.

To demonstrate the expected trends in experimental data, model predictions (Equations (8), (10), and (11)) for relevant parameters are shown in Figure 4. Specifically, the complex modulus $E^{*}$ is calculated for radii ranging from $10^{-6}$ to $10^{-3} \mathrm{~m}$ for a $6.0 \mu \mathrm{M}$ solution of $\mathrm{C}_{12} \mathrm{E}_{8}$.

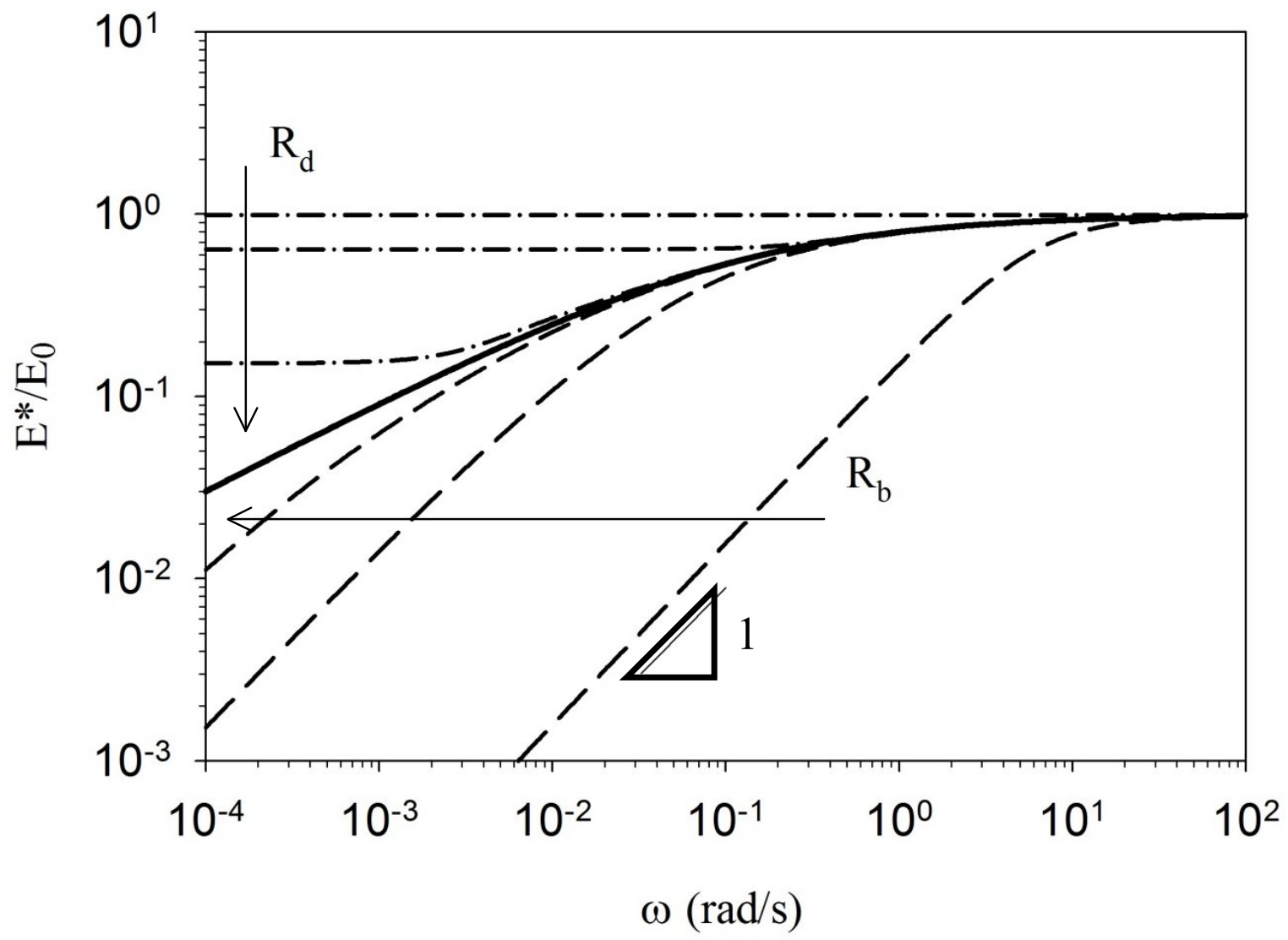

Figure 4. Normalized elasticity $\left(\mathrm{E}^{*} / \mathrm{E}_{0}\right)$ versus frequency $(\omega)$ using Equations (8) (planar, solid line), (10) (drop, dash-dot line), and (11) (bubble, dashed line) for $\mathrm{R}_{\mathrm{d}}$ and $\mathrm{R}_{\mathrm{b}}=1 \mathrm{~mm}, 0.1 \mathrm{~mm}$, and $1 \mu \mathrm{m}$. Arrows indicate direction of increasing radius. The terminal slope of 1 for the $R_{b}=1 \mu \mathrm{m}$ case is shown as well. $\mathrm{C}=6.0 \mu \mathrm{M}$ for all cases. 
Figure 4 shows the normalized elasticity as a function of frequency for a series of drops and bubbles of varying radii, as well as the planar model prediction which is a limiting case for both configurations. For each set of calculations, $E^{*} / \mathrm{E}_{0}$ increases with frequency up to the limiting value of the Gibbs elasticity at higher frequencies (where $\mathrm{E}^{*} / \mathrm{E}_{0}=1$ ). Just as in Figure 3, the predictions of $\mathrm{E}^{*}(\omega)$ for the drop are higher than the planar case, and the low frequency plateau of $E^{*}(\omega)$ increases with decreasing radius. In fact, for a drop radius of 1 micron, there appears to be no frequency dependence at all. For these higher values of curvature, the Gibbs modulus is sampled at all frequencies. For the bubble case, $E^{*}(\omega)$ decreases with decreasing radius and there is no low frequency plateau. Instead, as the bubble size decreases, the breadth of the terminal region at low frequencies increases.

Both the planar and bubble cases have a bulk concentration that is constant far away from the interface. In the case of a drop, this is often not the case and a limited supply of surfactant is available, making surfactant depletion an important consideration. ${ }^{[40]}$ Changes in the area therefore result in changes to the bulk concentration, which alters the lengthscale over which diffusion occurs. However, since this lengthscale is limited by the radius of the drop, the transport lengthscale (and by extension the diffusion timescale) becomes fixed. Thus at lower frequency oscillations, the elastic response approaches a limiting plateau due to the fact that the lengthscale of diffusion to the interface is limited by the drop size. This effect can be seen in the low-frequency pendant drop oscillatory data in the literature; for example, in [42], the data is explained by using the equation 10 and setting the drop radius between 0.25 and $1 \mathrm{~mm}$. To quantify the effect of curvature on the measurement of modulus, the low-frequency limit is taken for the modulus given in Equation (10),

$$
\lim _{\omega \rightarrow 0}\left(\frac{E_{D} *}{E_{0}}\right)=\frac{1}{1+\left(\frac{d C}{d \Gamma}\right) \frac{R}{2}},
$$

which gives the low-frequency plateau value of the elasticity as a function of the radius and surfactant isotherm. As shown in Figure (4), the planar and drop values of the moduli are the same above an isotherm and curvature dependent value of frequency, which we denote $\omega_{\mathrm{d}}$. Below this value, the magnitude of the modulus will deviate from its actual value by as much as the expression given in 
Equation (12). To quantify the value of $\omega_{\mathrm{d}}$, Equations (8) is solved the for frequency that corresponds to the modulus predicted in Equation (12). This assumes that the value of frequency at which the planar and drop solution deviate is approximately given by the point at which the low frequency plateau is reached. After solving for this frequency, an expression for $\omega_{d}$ is found that shows the isotherm and curvature dependence,

$$
\omega_{d}=\frac{2 D\left(\frac{d C}{d \Gamma}\right)^{2}}{\left(\left(1+2 R\left(\frac{d C}{d \Gamma}\right)+\frac{R^{2}}{2}\left(\frac{d C}{d \Gamma}\right)^{2}\right)^{1 / 2}-1\right)^{2}}
$$

Equation (13) provides the frequency, $\omega_{d}$, below which the planar assumption inherent in most pendant drop analysis is strongly influenced by curvature and likely to give erroneous results for transport and elasticity. This frequency is a function of the isotherm and radius, and provides a simple tool for assessing the impact of depletion on pendant drop experiments. Note that the natural lengthscale that arises in this express, $R(d C / d \Gamma)$, is dependent on both the curvature of the interface and the details of the isotherm. So the validity of the planar assumption is dependent on the nature of the surfactant and needs to be assessed for each system given an isotherm. These criteria are tested knowing the transport and isotherm properties of the surfactant.

For diffusion-limited surfactant transport to bubbles, a scaling argument developed by Alvarez et al. reveals the impact of curvature and transport on interfacial tension dynamics to an initially clean, stationary interface. ${ }^{[19]}$ Timescales for diffusion to a sphere are compared to timescales for diffusion to a planar interface, and the authors show that by decreasing bubble radius, diffusion timescales are drastically reduced. To evaluate the impact of curvature on the elasticity measurement, the timescales of both the planar and spherical cases are compared as a function of the lengthscale of the measurement. In the case of the oscillating bubble, the relevant lengthscales in the problem are the radius and the local 
changes in the depletion length, $\mathrm{d} \Gamma / \mathrm{dC}$. The ratio of the spherical to planar timescales is a function of the radius and $\mathrm{d} \Gamma / \mathrm{dC}$,

$$
\frac{\tau_{s}}{\tau_{p}}=f\left(R, \frac{d C}{d \Gamma}\right),
$$

where $\tau_{\mathrm{s}}$ is the characteristic timescale for the spherical problem, and $\tau_{\mathrm{p}}$ is the characteristic timescale for the planar problem (equivalent to the inverse of $\omega_{\mathrm{LV}}$ ). Because the bubble elasticity in Equation (11) is nonlinear with respect to both the frequency and radius, it is impossible to collapse the bubble data in Figure 4 onto a single curve with a simple scaling relationship. Instead, a single normalized elasticity is selected, and the resulting frequency of the planar and bubble models is extracted. This frequency corresponds to the inverse of the diffusion transport timescale for the problem. An appropriate choice for the elasticity is the value of $E^{*} / E_{0}$ which arises from the planar elasticity model in Equation (8) using the normalized frequency $\omega / \omega_{\mathrm{LV}}=1$. This results in a value of $\mathrm{E}^{*} / \mathrm{E}_{0}=0.54$. By solving Equation (11) for the frequency at which $\mathrm{E}^{*} / \mathrm{E}_{0}=0.54$, the frequency $\omega=\omega_{\mathrm{s}}$ is determined. The frequency $\omega_{\mathrm{s}}$ is inversely proportional to the diffusion timescale for the spherical problem. Performing this calculation for several values of $\mathrm{R}$ and $\mathrm{dC} / \mathrm{d} \Gamma$ yields a set of $\tau_{\mathrm{s}} / \tau_{\mathrm{p}}$ values corresponding to different normalized lengthscales.

To verify this analysis, we vary both bubble radius and surfactant concentration and perform experiments as well as simulations. Oscillatory $E^{*}(\omega)$ measurements are performed on the microtensiometer with a capillary radius of $42 \mu \mathrm{m}$ over a frequency range of $0.02-0.42 \mathrm{~Hz}$ for concentrations at $0.59,0.68,2.5,3.1$, and $6.0 \mu \mathrm{M} \mathrm{C}_{12} \mathrm{E}_{8}$. To calculate the timescale for the transport, $\tau_{\mathrm{s}}$ is determined by interpolating the frequency data near $E^{*} / E_{0}=0.54$. The planar timescale for each concentration, $\tau_{\mathrm{p}}$, is determined using Equation (9). Simulations are performed by solving Equations (1) (3) under the conditions of a sinusoidal deformation in bubble area for $\mathrm{C}=0.32 \mu \mathrm{M}$ to $0.1 \mathrm{mM} \mathrm{C}_{12} \mathrm{E}_{8}$, with $\mathrm{R}=42 \mu \mathrm{m}$ and over a frequency range of $10^{-7}$ to $10^{2} \mathrm{~Hz}$. In these calculations, the amplitude of the area deformation is $5 \%$, and the oscillation lasted seven total periods. The elasticity is determined by fitting both the surface tension response and area deformation to sine waves, and calculating $\mathrm{E}^{*}(\omega)$. Figure 5 is a plot of $\tau_{\mathrm{s}} / \tau_{\mathrm{p}}$ versus $\mathrm{R}(\mathrm{dC} / \mathrm{d} \Gamma)$ for the scaling analysis performed using Equations (8) and (11), for the interpolated experimental data, and for the oscillatory simulations. Also plotted is the scaling analysis from Alvarez et al., ${ }^{[19]}$ as $\tau_{\mathrm{s}} / \tau_{\mathrm{p}}$ versus $\mathrm{R}(\mathrm{C} / \Gamma)$ (or the radius normalized by the depletion depth). 


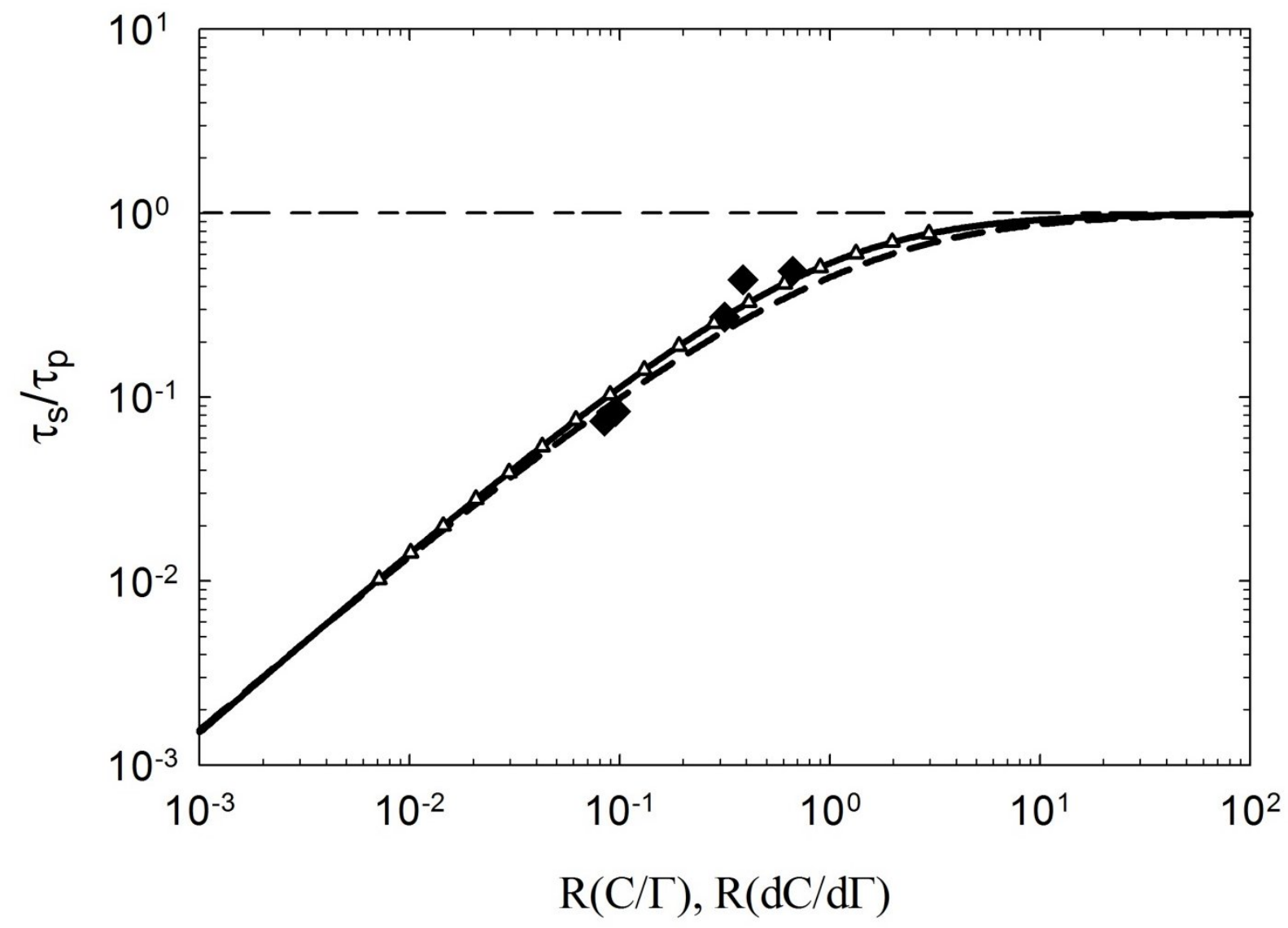

Figure 5. $\tau_{\mathrm{s}} / \tau_{\mathrm{p}}$ versus $\mathrm{R}(\mathrm{C} / \Gamma)$ (dashed line) from Alvarez et al. ${ }^{[19]}$ and $\tau_{\mathrm{s}} / \tau_{\mathrm{p}}$ versus $\mathrm{R}(\mathrm{dC} / \mathrm{d} \Gamma)$ determined using Equation (11) (solid line), determined from experiments $\left(\diamond, \mathrm{R}_{b}=42 \mu \mathrm{m}\right)$ for $\mathrm{C}=0.59,0.68,2.5$, 3.14, and $6.0 \mu \mathrm{M} \mathrm{C}_{12} \mathrm{E}_{8}$, and from simulations using Equations (1) - (3) $\left(\Delta, \mathrm{R}_{\mathrm{b}}=42 \mu \mathrm{m}\right)$ for $\mathrm{C}=0.32 \mu \mathrm{M}$ to $0.10 \mathrm{mM} \mathrm{C}_{12} \mathrm{E}_{8}$.

In Figure 5, all values of $\tau_{\mathrm{s}} / \tau_{\mathrm{p}}$ decrease with decreasing normalized lengthscale $\mathrm{R}(\mathrm{dC} / \mathrm{d} \Gamma)$ for elasticity measurements or $\mathrm{R}(\mathrm{C} / \Gamma)$ for the scaling analysis from Alvarez et al. ${ }^{[19]}$ For large values of the 
normalized lengthscale, $\tau_{\mathrm{s}} / \tau_{\mathrm{p}}$ approaches a value of 1 . Thus at large radius or high concentration, the interface responds as though it is planar. This demonstrates how the timescale of diffusive transport can be reduced by orders of magnitude simply by altering the curvature of the problem. This scaling analysis is inherently similar to that provided by Alvarez et al., ${ }^{[19]}$ with the exception that the appropriate length scale is $\mathrm{R}(\mathrm{C} / \Gamma)$, and the scaling is slightly lower for the range $\mathrm{R}(\mathrm{C} / \Gamma)=10^{-2}$ to $10^{1}$. This difference constitutes at most a $15 \%$ difference in values of $\tau_{\mathrm{s}} / \tau_{\mathrm{p}}$. The oscillatory curve is within $99 \%$ of planar behavior at a normalized length scale of 55, experimentally observed at either large radii or high concentrations. From Alvarez et al. ${ }^{[19]}$ the timescale reaches $99 \%$ of planar behavior at a normalized length scale of 100 . The limiting slope at small values of the normalized length scale is 1 for both curves on a $\log -\log$ scale.

There are important considerations when applying oscillatory deformations to a curved interface. For all of these analyses, small amplitude oscillations are assumed. A number of phenomena can give rise to nonlinear responses and higher harmonics, including nonlinearity in the interfacial constitutive relations, convection in the bulk near the interface, and induced Marangoni stresses along the interface. To prevent higher-order harmonics like those resulting from convection, ${ }^{[35]}$ it has been suggested that amplitudes should range from 1 to $5 \% .{ }^{[25]}$ Another consideration is the fact that any oscillating waveform applied to the area begins with an expansion (or contraction) and the impact of that initial "step" on the response of the interface needs to be considered. Any startup transients are amplified with higher amplitude oscillations.

To first demonstrate the effect of a large amplitude oscillation on the surface tension of the systems considered, a $12.5 \%$ amplitude and $\omega=1 \mathrm{~Hz}$ oscillation is simulated using Equations (1) - (3) on a pre-equilibrated $1 \mathrm{~mm}$ bubble with a bulk concentration of $6.0 \mu \mathrm{M} \mathrm{C} \mathrm{C}_{12} \mathrm{E}_{8}$. In this case a cosine waveform is applied which begins with an expansion of the interfacial area. For comparison, a 12.5\% step change in area is also applied to an interface. These results are plotted as the surface tension versus time in Figure 6. 


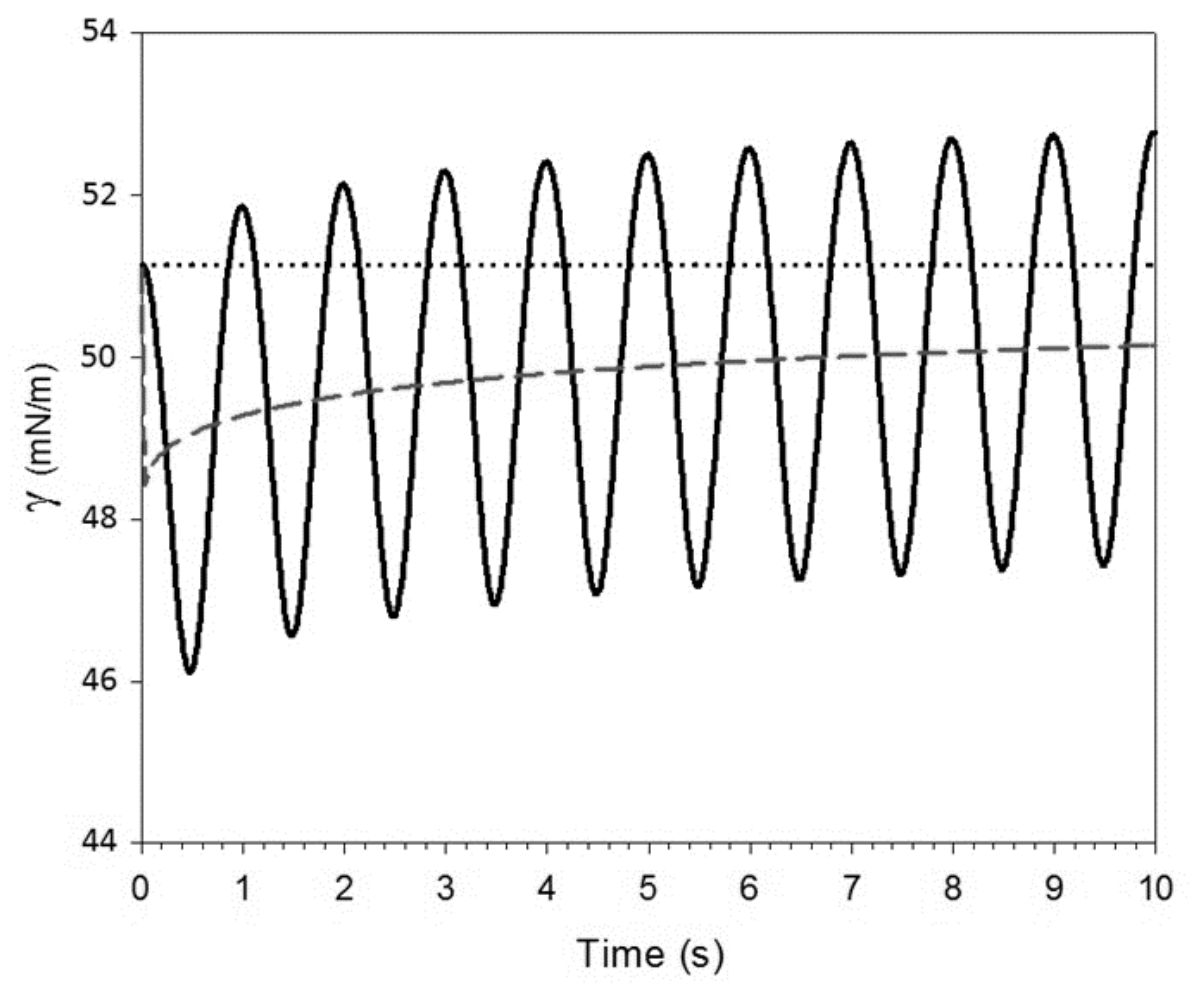

Figure 6. Surface tension, $\gamma$, versus time for a simulated $12.5 \%$ amplitude oscillation on a $1 \mathrm{~mm}$ bubble (solid line) at a bulk concentration of $6.0 \mu \mathrm{M} \mathrm{C}_{12} \mathrm{E}_{8}$, and simulation results for a step change equal to the amplitude of oscillation (dashed line) using Equations (1) - (3). The dotted line is the equilibrium value of the surface tension.

In Figure 6, the median surface tension for both the oscillation and step change initially decreases from $51.6 \mathrm{mN} / \mathrm{m}$ to $48.4 \mathrm{mN} / \mathrm{m}$, and then slowly increases toward the equilibrium value. The median value of the simulated oscillation follows the transient associated with the step change. Both simulations result in a sudden change in the median coverage of surfactant at the interface. This initial shock to the interface will recover while the oscillations are being analyzed and will impact the extracted elasticity.

The problem is lessened, but not eliminated, by using a sine wave (which begins with a contraction of half an amplitude). Two $12.5 \%$ amplitude oscillations are simulated again at $1 \mathrm{~Hz}$ on a 1 $\mathrm{mm}$ bubble with $\mathrm{C}=6.0 \mu \mathrm{M} \mathrm{C}_{12} \mathrm{E}_{8}$ (not shown). To calculate the elasticity decay, the peak and trough of each period in the surface tension of the sine wave is used to determine $\Delta \gamma$, while $\Delta A / A_{0}$ remains fixed. 
For this scenario, $\mathrm{E}^{*}$ begins higher than the expected $\mathrm{E}^{*}$ of $27.4 \mathrm{mN} / \mathrm{m}$ for both oscillation mode, and decays over time toward the true value. For the sine wave, the initial difference is $0.1 \mathrm{mN} / \mathrm{m}$, which is within error of most current devices and decays in about 10 seconds. For the cosine oscillation, $\mathrm{E}^{*}$ begins near 30.3, which is a measurable deviation from $27.4 \mathrm{mN} / \mathrm{m}$. The system requires nearly 1000 seconds to achieve the difference of $0.1 \mathrm{mN} / \mathrm{m}$. This "startup" effect is evident due to the fact that the isotherm is not linear over large deformations, and so some initial offset is incurred.

During a frequency sweep measurement of the elasticity, these startup effects are pronounced if insufficient time is allowed for the bubble or drop to regain the median equilibrium value of the surface tension. To demonstrate this point, a pendant drop cosine oscillation experiment is performed at $12.5 \%$ amplitude for a drop equilibrated at $51.6 \pm 0.1 \mathrm{mN} / \mathrm{m}$, indicating a depleted bulk solution of $6.0 \mu \mathrm{M} \mathrm{C}_{12} \mathrm{E}_{8}$ (the same for the drop in Figure 3). The frequency is varied from 0.1 to $1 \mathrm{~Hz}$. In this range, the planar equation for the elasticity converges with the drop equation (the minimum plateau frequency is calculated to be $5 \times 10^{-4} \mathrm{~Hz}$ ), so simulations are performed assuming a planar interface. For both experiments and simulations, the elasticity is determined by fitting a sine wave to the surface tension data after the first three oscillations. The elasticity is plotted as a function of frequency in Figure 7 for both the large amplitude simulation and data, and the pendant drop data and planar elasticity calculation from Figure 3 for comparison. This demonstrates the magnitude of the difference that will be observed in the measured modulus for a realistic system. At low frequencies, the difference is small, but it is significant at higher frequencies and highlights the danger of poorly planned experiments; both the decay of stresses from the initial area change in a cosine function and the impact of amplitude. 


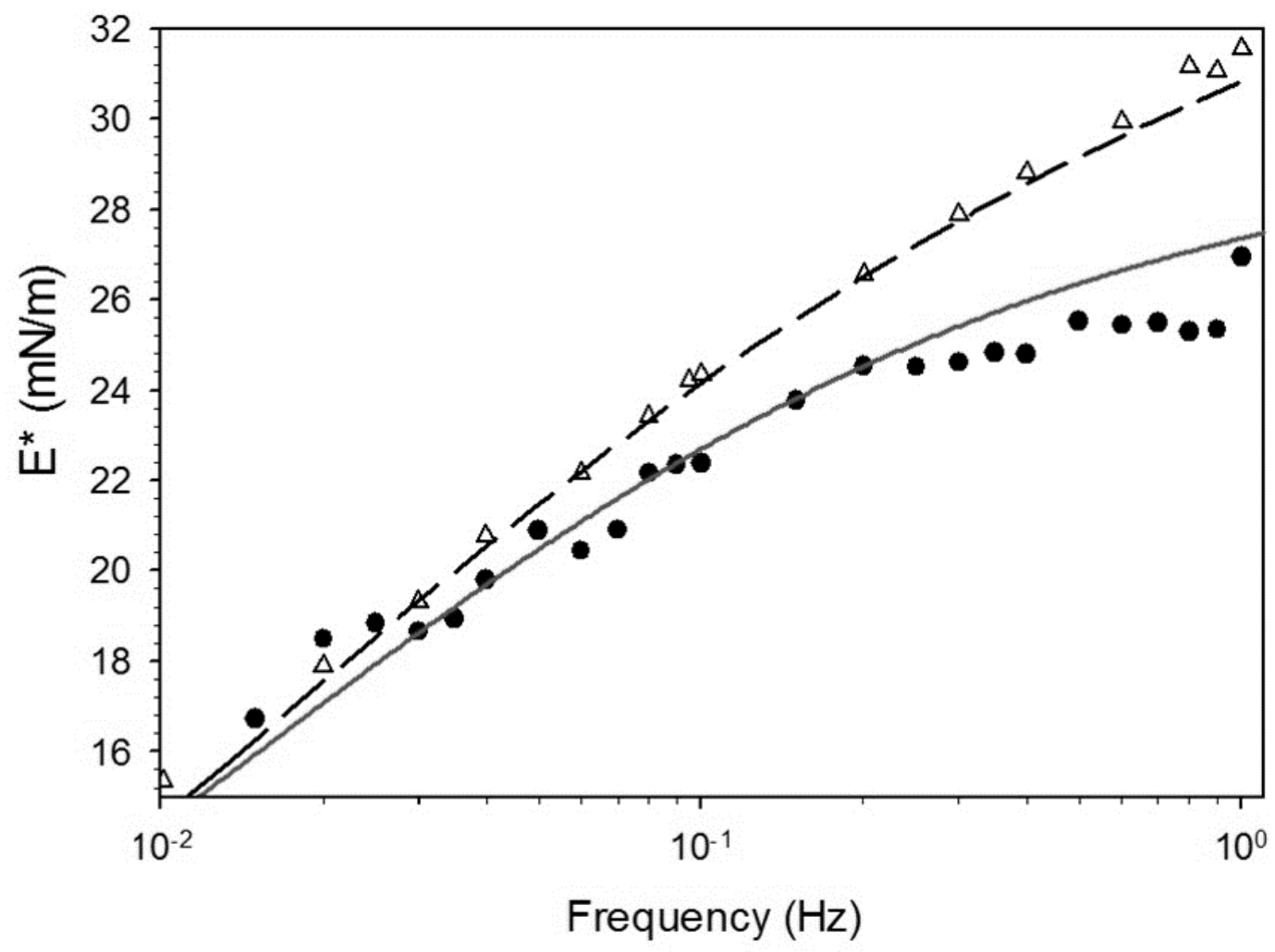

Figure 7. $E^{*}$ versus frequency for $\mathrm{C}_{12} \mathrm{E}_{8}$ at $\mathrm{C}=6.0 \mu \mathrm{M}$. Symbols are data from a $12.5 \%$ cosine oscillation of the drop area $(\Delta)$, and a $5 \%$ amplitude sine oscillation $(\bullet)$. The dashed line corresponds to simulation results, and the solid line corresponds to calculations using Equation (10).

\section{Discussion}

Many studies in the literature utilize the planar solution to describe oscillatory behavior of diffusion-limited surfactant systems, and fit complex transport models to extract parameters such as diffusion coefficients. In fact, there are no fitting parameters for the model if the bulk diffusion coefficient and the isotherm are known. The onset of curvature, depletion and other experimental effects can easily be misinterpreted as unphysical variations in the diffusion coefficient.

In general, the largest differences between the planar model and the models that utilize curvature are seen at slow rates of deformation of the interface. Figures $2-4$ demonstrate this phenomenon for the 
surfactant $\mathrm{C}_{12} \mathrm{E}_{8}$, as clear deviations are observed between calculated planar expansions and oscillations, and experimental data for pendant drops and microbubbles.

To determine the magnitude of this effective shift in timescales, an analysis was performed similar to what has been shown previously for diffusion-limited surfactant systems. ${ }^{[19]}$ Figure 5 depicts the transition from a planar transport timescale toward the faster spherical timescale as the lengthscale of the problem is reduced. In the case of dilatational elasticity measurements, that lengthscale is $R d C / d \Gamma$, however in diffusion to an initially clean, stationary interface the lengthscale is $R C / \Gamma$. While the two timescales are similar, it is notable that the oscillatory timescale applies to concentrated surfactant systems where it is impractical to achieve an initially clean interface in the observation timescales. In this case, the scaling analysis presented here shows that a dilatational elasticity experiment can be used to probe the dominant transport mechanism. Thus, measurement of the dilatational elasticity of a surfactant laden interface in concentrated solution serves as a complementary technique to the conventional dynamic surface tension measured at an initially clean interface for dilute solutions. The dominant transport mechanism can be elucidated using Figure 5. Systems with mixed dependence on reaction kinetics and diffusion, or systems that are entirely limited by reaction kinetics, exhibit characteristic timescales larger than those expected for diffusion-limited systems. ${ }^{[19,21]}$ In those instances, the measured timescale of the experiment would lie above that predicted for diffusion-limited transport to a sphere. By incorporating the kinetics of adsorption into the dilatational elasticity measurement, kinetic rate constants can be measured (such equations, while not used here, are derived in part in reference [35]).

Large amplitudes and poor startup conditions in oscillatory measurements of dilatational modulus can lead to long startup transients and erroneous elasticity values. Simple estimates suggest that 5\% oscillation amplitude can be considered "small amplitude" for $\mathrm{C}_{12} \mathrm{E}_{8}$. However, using the isotherm of the surfactant, a simple approximation of the amplitude dependence on the value of $\mathrm{dC} / \mathrm{d} \Gamma$ can be performed by calculating the derivative of Equation (4), and determining $\mathrm{dC} / \mathrm{d} \Gamma$ for values of $\Gamma \pm \delta \Gamma$, where $\delta$ is the amplitude of the experiment. For $\mathrm{C}_{12} \mathrm{E}_{8}$ at a concentration of $6.0 \mu \mathrm{M}$, a five percent area change, without incorporating transport, corresponds to a three percent change in $\mathrm{dC} / \mathrm{d} \Gamma$. The resulting difference in the 
Gibbs elasticity is $0.02 \mathrm{mN} / \mathrm{m}$, which falls well within the error of any pendant drop, pendant bubble, or microtensiometer device. This confirms that a 5\% amplitude acceptable for experiments on this system.

In Figures 6 and 7, the impact of poorly designed oscillations is shown. The use of a cosine function can lead to an effective step change in the median area. In one publication, the authors account for this by waiting 30 minutes to an hour after starting an oscillation before measurement, but this is not universally done in the literature. ${ }^{[25]}$ The microtensiometer accounts for this by oscillating the pressure within the bubble, which imparts a sinusoidal deformation of the surface tension, limiting startup effects. In Figure 7, a large amplitude cosine oscillation is utilized in a pendant-drop setup, demonstrating the effect on experimental results. The deviations arise simply because the isotherm is non-linear over large amplitudes, and startup effects induce a step change in the median area. It should also be noted that the cosine oscillations are performed in the direction of compression; large amplitude cosine oscillations in the expansion direction would yield a reduced elasticity, as the median surface coverage of surfactant would be lower.

\section{Conclusions}

The impact of curvature and depletion on the dilatational modulus of a surfactant-laden interface is examined. Experiments for a wide range of conditions, including geometry, area rates of change, and types of deformation (constant ramp versus oscillation), all agree well with diffusion-limited transport models using the molecular value of diffusivity and correctly accounting for all of these effects.

Geometry and depletion effects on transport are more pronounced at low deformation rates and frequencies. Curved bubbles exhibit lower elasticity at lower frequencies, which implies that low frequencies/rates can be used to probe transport parameters. For example, in pendant drop measurements, the dilatational modulus reaches a plateau at low frequency as depletion causes the relevant lengthscale to cut off at the drop radius. The roll off frequency to the low frequency plateau thus provides an estimate of conditions at which depletion effects become significant.

Characteristic timescales (or inverse characteristic frequencies) associated with dilatational modulus measurements scale with drop radius and concentration in a similar way to those for dynamic surface tension measured at an initially clean, stationary bubble interface. 


\section{Acknowledgements}

The authors acknowledge Dr. Anthony Kotula (CMU) and both Timothy Koehler and Chris Brotherton (Sandia) for useful discussions. Sandia National Laboratories is a multi-program laboratory managed and operated by Sandia Corporation, a wholly owned subsidiary of Lockheed Martin Corporation, for the U.S. Department of Energy's National Nuclear Security Administration under contract DE-AC04-94AL85000.

\section{Works Cited}

1. $\quad$ S.Y. Lin, K. McKeigue, C. Maldarelli, AIChE J., 36 (1990) 1785.

2. S.H. Anastasiadis, J.K. Chen, J.T. Koberstein, A.F. Siegel, J.E. Sohn, J.A. Emerson, Journal of Colloid and Interface Science, 119 (1987) 55.

3. M. Hoorfar, A.W. Neumann, Advances in Colloid and Interface Science, 121 (2006) 25.

4. $\quad$ N.J. Alvarez, L.M. Walker, S.L. Anna, Journal of Colloid and Interface Science, 333 (2009) 557.

5. G. Loglio, E. Rillaerts, P. Joos, Colloid and Polymer Science, 259 (1981) 1221.

6. $\quad$ N.F. Djabbarah, D.T. Wasan, Chemical Engineering Science, 37 (1982) 175.

7. C.A. Macleod, C.J. Radke, Journal of Colloid and Interface Science, 166 (1994) 73.

8. D.O. Johnson, K.J. Stebe, Journal of Colloid and Interface Science, 168 (1994) 21.

9. H. Fruhner, K.D. Wantke, Colloids and Surfaces A: Physicochemical and Engineering Aspects, 114 (1996) 53.

10. J.F. Baret, Journal of Colloid and Interface Science, 30 (1969) 1.

11. F. Ravera, Liggieri, L., Steinchen, A., Journal of Colloid and Interface Science, 156 (1993) 109.

12. R. Nagarajan, D.T. Wasan, Journal of Colloid and Interface Science, 159 (1993) 164.

13. J. Vanhunsel, P. Joos, Colloids and Surfaces, 24 (1987) 139.

14. J.K. Ferri, S.Y. Lin, K.J. Stebe, Journal of Colloid and Interface Science, 241 (2001) 154.

15. V.G. Levich, Physicochemical hydrodynamics : Veniam G. Levich; translated by Scripta Technica Inc. Prentice-Hall international series in the physical and chemical engineering sciences. 1962, Englewood Cliffs, N.J.: Prentice-Hall.

16. D. Ilkovič, Collect. Czech. Chem. Commun., 6 (1934) 498.

17. N.J. Alvarez, W. Lee, L.M. Walker, S.L. Anna, Journal of Colloid and Interface Science, 355 (2011) 231.

18. N.J. Alvarez, L.M. Walker, S.L. Anna, Langmuir, 26 (2010) 13310.

19. N.J. Alvarez, L.M. Walker, S.L. Anna, Physical Review E, 82 (2010).

20. R.Y. Tsay, S.Y. Lin, L.W. Lin, S.I. Chen, Langmuir, 13 (1997) 3191.

21. R. Miller, J.K. Ferri, A. Javadi, J. Kragel, N. Mucic, R. Wustneck, Colloid and Polymer Science, 288 (2010) 937.

22. F. Ravera, G. Loglio, V.I. Kovalchuk, Current Opinion in Colloid \& Interface Science, 15 (2010) 217.

23. D. Langevin, F. Monroy, Current Opinion in Colloid \& Interface Science, 15 (2010) 283.

24. D.O. Johnson, K.J. Stebe, Colloids and Surfaces a-Physicochemical and Engineering Aspects, 114 (1996) 41. 
25. D.O. Johnson, K.J. Stebe, Journal of Colloid and Interface Science, 182 (1996) 526.

26. N. Duerr-Auster, R. Gunde, E.J. Windhab, Langmuir, 24 (2008) 12282.

27. E.M. Freer, K.S. Yim, G.G. Fuller, C.J. Radke, J. Phys. Chem. B, 108 (2004) 3835.

28. L.S. Jourdain, C. Schmitt, M.E. Leser, B.S. Murray, E. Dickinson, Langmuir, 25 (2009) 10026.

29. B.A. Noskov, A.A. Mikhailovskaya, S.Y. Lin, G. Loglio, R. Miller, Langmuir, 26 (2010) 17225.

30. A.A. Perez, C.C. Sanchez, J.M. Rodriguez Patino, A.C. Rubiolo, L.G. Santiago, Colloids and Surfaces B-Biointerfaces, 81 (2010) 50.

31. J.T. Petkov, T.D. Gurkov, B.E. Campbell, R.P. Borwankar, Langmuir, 16 (2000) 3703.

32. J. Lucassen, M. Van Den Tempel, Chemical Engineering Science, 27 (1972) 1283.

33. I.B. Ivanov, K.D. Danov, K.P. VAnanthapadmanabhan, A. Lips, Advances in Colloid and Interface Science, 114-115 (2005) 61.

34. J.W. Gibbs, The collected works of J. W. Gibbs. 1902: Longmans, Green, and Co.

35. P. Joos, Dynamic Surface Phenomena, Eds. V.B. Fainerman, G. Loglio, EH Lucassen-Reynders, R. Miller and P. Petrov. 1999, The Netherlands: VSP BV.

36. E.V. Aksenenko, V.I. Kovalchuk, V.B. Fainerman, R. Miller, Advances in Colloid and Interface Science, 122 (2006) 57.

37. A. Dan, G. Gochev, J. Kraegel, E.V. Aksenenko, V.B. Fainerman, R. Miller, Current Opinion in Colloid \& Interface Science, 18 (2013) 302.

38. C. Kotsmar, V. Pradines, V.S. Alahverdjieva, E.V. Aksenenko, V.B. Fainerman, V.I. Kovalchuk, J. Kragel, M.E. Leser, B.A. Noskov, R. Miller, Advances in Colloid and Interface Science, 150 (2009) 41.

39. S. Karakashev, R. Tsekov, E.D. Manev, A.V. Nguyen, Colloids and Surfaces a-Physicochemical and Engineering Aspects, 369 (2010) 136.

40. $\quad$ N.J. Alvarez, L.M. Walker, S.L. Anna, Soft Matter, 8 (2012) 8917.

41. N.J. Alvarez, D.R. Vogus, L.M. Walker, S.L. Anna, Journal of Colloid and Interface Science, 372 (2012) 183.

42. C. Stubenrauch, R. Miller, J. Phys. Chem. B, 108 (2004) 6412.

43. V.B. Fainerman, S.A. Zholob, J.T. Petkov, R. Miller, Colloids and Surfaces A-Physicochemical and Engineering Aspects, 323 (2008) 56. 